\title{
NUMERICAL SIMULATION OF FLUID-STRUCTURE INTERACTION FOR THIN FLAT DELTA WING AT TRANSONIC SPEED BASED ON OPEN-SOURCE SOFTWARE
}

\author{
YUSUKE TAKAHASHI* \\ *Hokkaido University \\ Kita 13 Nishi 8, Kita-ku, Sapporo, Hokkaido 060-8628, Japan \\ e-mail: ytakahashi@eng.hokudai.ac.jp
}

Key words: Coupled Problems, Fluid-Structure Interaction, Transonic Flutter, LimitCycle Oscillation, Open-Source Software

\begin{abstract}
The flutter of a thin flat-delta wing at transonic speed was investigated by fluid-structure interaction (FSI) analysis, which is based on the open-source software. The analysis model was composed of fluid solver SU2, structure solver CalculiX, and coupler preCICE library. The FSI coupling of both solvers was performed in a partitioned approach. The software and libraries were built on a cloud computing system in Hokkaido University. It was found that self-induced oscillation of the delta wing is induced by the shock wave and elastic deformation in the transonic regime. The primary frequency of the oscillation was approximately $20 \mathrm{~Hz}$ for all speeds considered herein. However, the eigenfrequencies of the present condition of the delta wing, which are 9.62, 36.69, 51.22 , 88.94, etc., did not correspond to the oscillation frequency. The phase delay of aerodynamic force for the deformation of the delta wing appeared in the oscillation. It was indicated that the oscillation is amplified by the aerodynamic force at the low deformation phase, and that is attenuated at a high deformation phase. In other words, in one cycle, the wing is in an unstable state by receiving the energy from fluid flow at the low deformation and is in a stable state by passing the energy to fluid flow at high deformation. When this energy transfer is equilibrated, the oscillation reaches the limit cycle. It was found that this behavior of the delta wing at the transonic speed is attributed to the shock wave and elastic deformation, i.e., coupling between flow and structure.
\end{abstract}

\section{INTRODUCTION}

Fluid-structure interaction (FSI) is a significant topic for mechanical engineering and aerospace engineering. Examples of FSI problems in high enthalpy flows which appear in rocket launching and atmospheric entry include aeroelastic problems in the fins or 
body of rockets, coupling of aerodynamic forces and membrane aeroshells in inflatable reentry vehicles $[1,2]$, and behavior of parachute during descent. These are issues that require careful consideration in design and development because they can cause significant problems in some cases.

Estimations using wind tunnel facilities is important for FSI problems. However, it is difficult to reproduce actual conditions because the scale law can not always be applied. Numerical analysis removes this limitation and is expected to play a certain role in research and development. Single-physics problem such as flow and structure have been studied extensively, and the software for analysis has become quite sophisticated. On the other hand, however, there is still not much software available to deal with multiphysics problems such as FSI analysis. This is especially notable under limited conditions such as high enthalpy flows.

In recent years, many open-source software (OSS) have developed. OSS is becoming possible to solve various problems with a high degree of freedom. For example, SU2 [3], a compressible fluid analysis software, and CalculiX [4], a finite element analysis software, are ones of OSS. These are basically software for single-physics problems and have been used mainly for that purpose. Recently, an OSS coupling library called preCICE [5] has been developed, which enables to couple multiple software by simply preparing a small

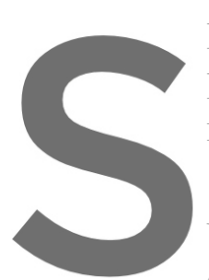
program called an ackapter.

preCICE are already flows

Transonic flutter which is a combinatio destructive oscillation
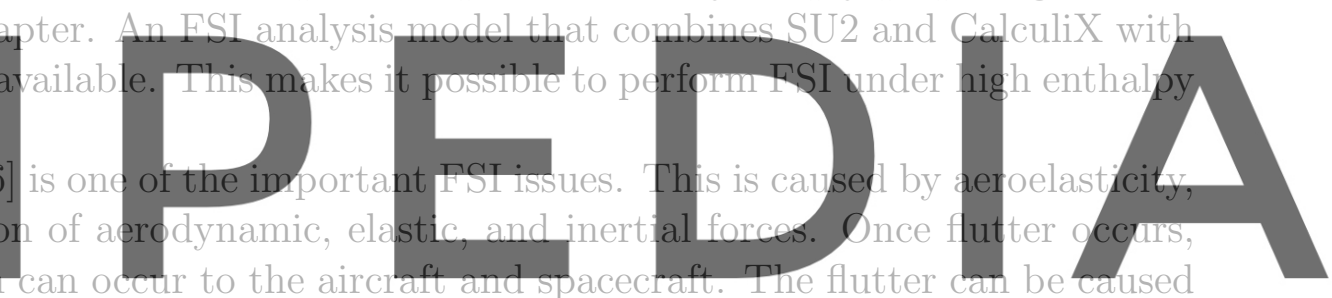

by the transonic dip, which tends to cause instability in the transonic region, and by the

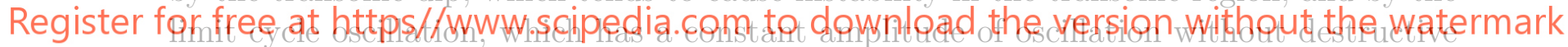

oscillation. Unsteady aerodynamic forces play a major role in flutter, and an appropriate structure analysis model is necessary to evaluate the elastic forces. The coupling between the aerodynamic and elastic problems plays an important role in the analysis, which requires large computational resources.

In recent years, it has become easier to use relatively-large computational resources, and FSI analysis software for high enthalpy flows has been developed. In this paper, the results of the FSI analysis of flutter in the transonic region using these software are reported.

\section{FSI MODELING}

The present FSI analysis model consists of SU2 fluid analysis software for unstructured grids as a flow solver, CalculiX finite element analysis software as a structure solver, and preCICE library as a coupler. The software with preCICE adapters [7] is available on Github. 


\subsection{Analysis Object}

A thin flat delta wing with an isosceles triangle shape is placed in a freestream transonic flow with no angle of attack. As shown in Fig. 1, the delta wing has $40 \mathrm{~mm}$ sides and 1 mm thickness. The fixed points are the isosceles connecting the vertices "AC" shown in Fig. 1.

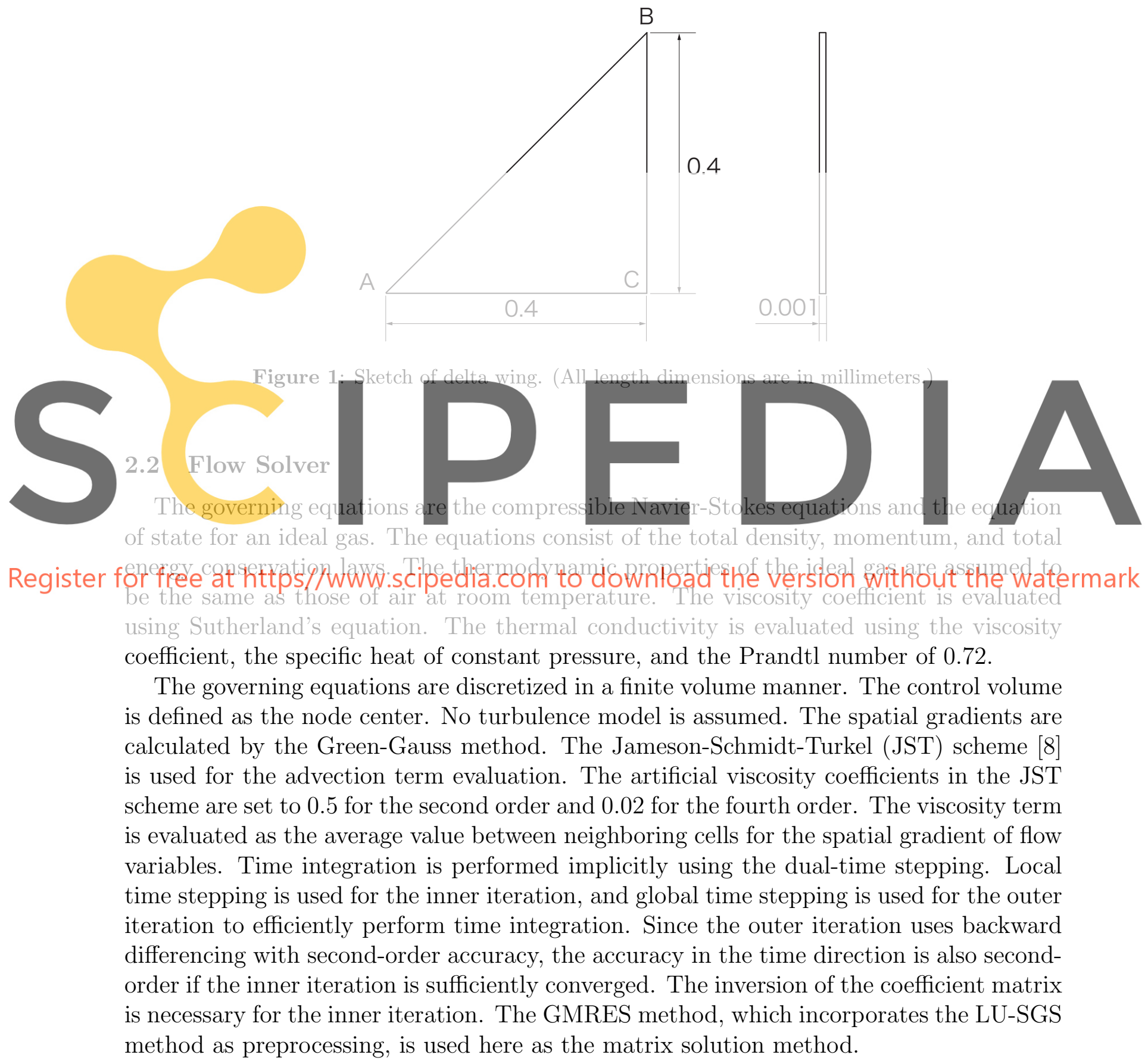


SU2-v6.0.0, which is a version installing preCICE adapter, is used as the flow solver, which supports unstructured grids data and has high shape reproducibility. The parallel computation is performed using the domain decomposition method and the message passing interface (MPI). Domain decomposition is performed by ParMETIS [9], which is a standard parallel method for flow simulations on large scale computers and can be used on various computers.

\subsection{Structure Solver}

The structural dynamics equations are described based on the principle of virtual work . Since the temperature change inside the object is not considered here, the momentum equilibrium of the displacement field is obtained. The equation is discretized by small elements and consists of the displacement in each element, the element stiffness matrix, the element load vector, and the element mass matrix. The element mass matrix is calculated by using the shape function used in the formulation of the element stiffness matrix.

By defining the displacement vector, the stiffness equation consisting of the stiffness matrix, the mass matrix, and the load vector can be obtained. The node displacements can be obtained by solving this equation under certain constraint conditions. In addition,

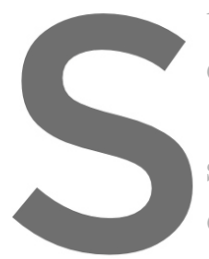
the reaction force at element are calculatec Calculix v2.15, which solver. A three-dimensional cretize the object.
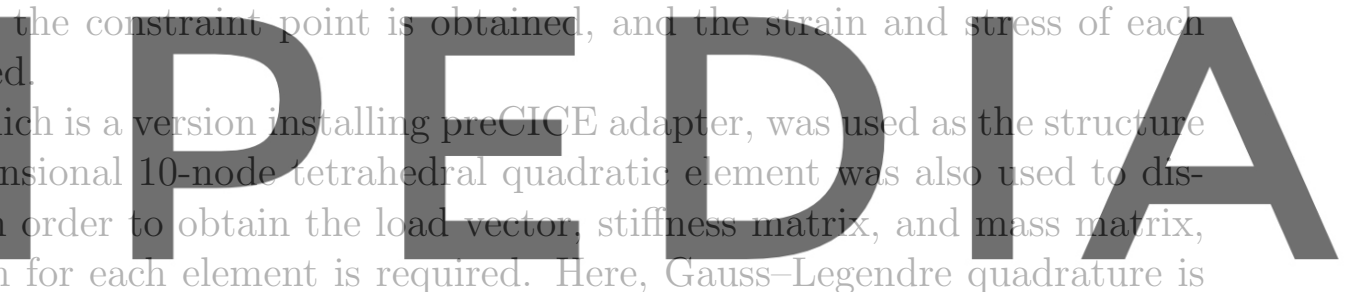
numerical integration for each element is required. Here, Gauss-Legendre quadrature is Register for free at https/fwww scipedia.con the download the version without the watermark order accurate and unconditionally stable when the parameter $\alpha \in-1 / 3,0]$, which is set $\alpha=-1 / 3$ here. In addition, the analysis is performed in geometrically nonlinear mode to handle large deformations. To do this, iterative calculations are required, which increases the amount of calculation. The material properties are assumed to be linear.

\subsection{Coupling Method}

The coupled method employed in preCICE is classified as a partitioned coupling, i.e., weakly coupling. Flow simulation and structure simulation are performed by the independent software mentioned above. Data for the coupled interface is passed between each other via the preCICE library. At this time, the continuity and equilibrium conditions between data are assumed to exist at the coupled interface.

The present coupled interface is the entire surface of the flat wing. The physical variables passed at the coupled interface are aerodynamic force on the flow solver and displacement on the structure solver. The computational grids at the coupled interface are necessarily not the same for both the flow and structure solvers. Even if the two 
computational grids are different, data can be mapped and interpolated appropriately. Nearest-projection mapping is used here for the interpolation.

Data communication between the flow and structure solvers is performed using TCP/IP sockets.

To satisfy the continuity and equilibrium conditions, an implicit coupling scheme is adopted. That iterates through the fluid-structure partitioned system in a single time step. In the case of an implicit coupling scheme, the coupling can be performed more efficiently by introducing an appropriate convergence acceleration scheme or stabilization scheme in the iterations. Here, the interface quasi-Newton method [11] is adopted.

PreCICE v1.61, which implements these methods, is adopted.

\subsection{Computational Conditions}

Figure 2 shows the computational grids for the flow simulation. A flat delta wing is set to be placed in a freestream flow. The reservoir pressure, temperature, and Mach number are given as freestream conditions. The freestream parameters are determined from the equation of the isentropic process. In this study, Mach numbers of 0.9 and 1.1 are used for the cases of reservoir pressures of 1000, 5000, 8000, and $10000 \mathrm{~Pa}$, and reservoir temperatures of $300 \mathrm{~K}$ are used. When the characteristic length is $0.4 \mathrm{~m}$, the Reynolds

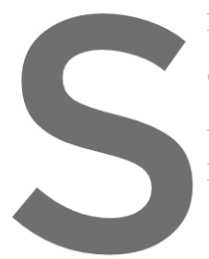
number is of the order assumed to be fixed at All computational g is $2,111,38$ and the
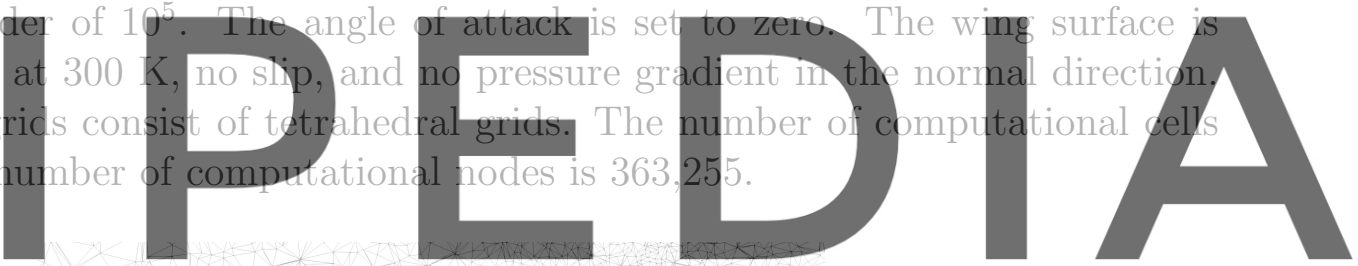

Register for free at https//www.scipedia.com to download the version without the watermark

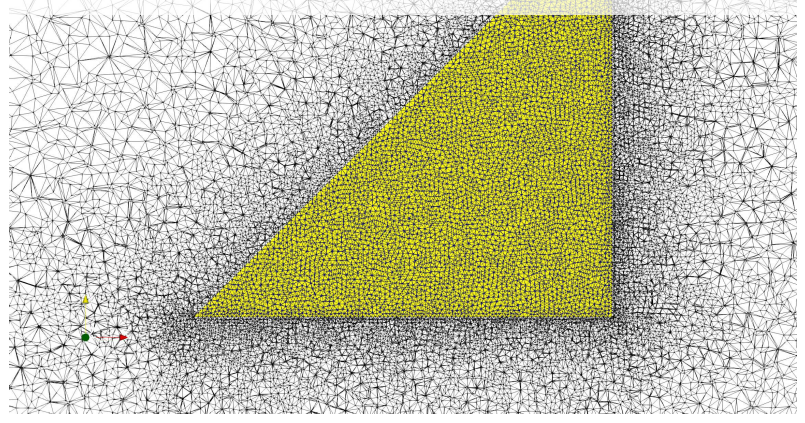

Figure 2: Computational grids for flow solver SU2.

The computational mesh used for the structure simulation is shown in Fig. 3. This mesh also consists of tetrahedral shapes, but quadratic elements are considered to improve the accuracy. The number of nodes is 154,932 . One side of the delta wing is set as a 
fixed boundary. All the others are free boundaries and coupled interfaces. The wing is assumed to be composed of a single material. The mechanical properties of the material are assumed to be similar to those of an aluminum alloy, with a Young's modulus of 74 GPa, a Poisson's ratio of 0.355 , and a density of $2800 \mathrm{~kg} / \mathrm{m}^{3}$.

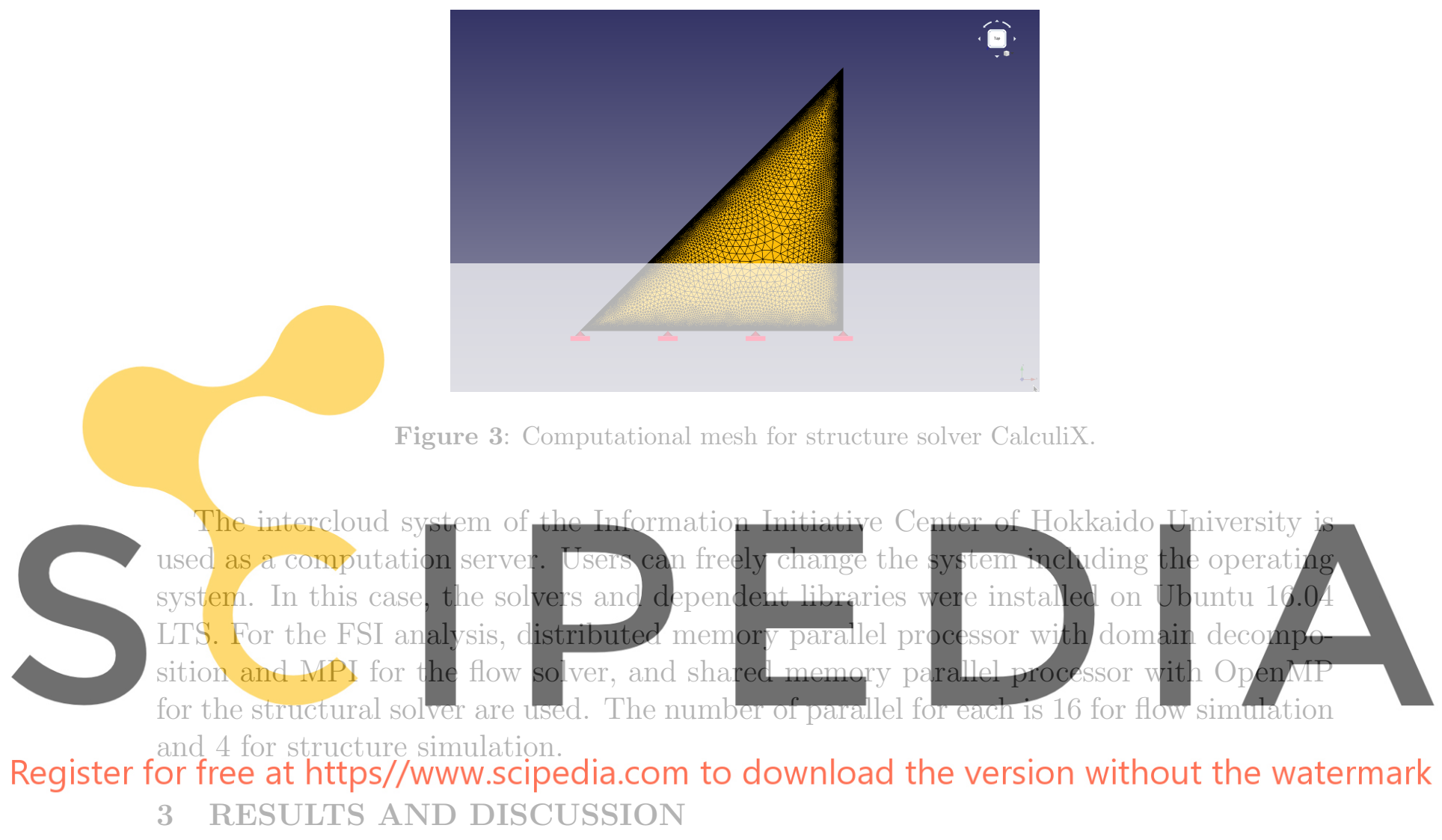

The time histories of the displacements were investigated by using probe technique into one of the three vertices of the delta wing that do not contain a fixed boundary, i.e., vertex "B" in Fig. 1. The displacement histories in the z-direction for freestream Mach numbers of 0.9 and 1.1 is shown in Fig. 4 for each dynamic pressure condition. At a high dynamic pressure of $8000 \mathrm{~Pa}$, self-induced oscillations are observed. On the other hand, at low dynamic pressures of 1000 and $5000 \mathrm{~Pa}$, small oscillations occur, but large self-induced oscillations are not caused. At Mach number 0.9 , the self-induced oscillation settles down to a constant amplitude and the limit cycle is confirmed, whereas at Mach number of 1.1, the displacement still tends to increase in this period. The self-induced oscillation with some large amplitude indicates the existence of a certain Mach number region and dynamic pressure region.

Fast Fourier transform (FFT) was performed on the displacement histories from 0 to $0.5 \mathrm{~s}$. The Hamming window function was used. Figure 5 shows the condition of Mach number 1.1 and dynamic pressure $10000 \mathrm{~Pa}$. In this condition, a peak at $64 \mathrm{~Hz}$ can be 


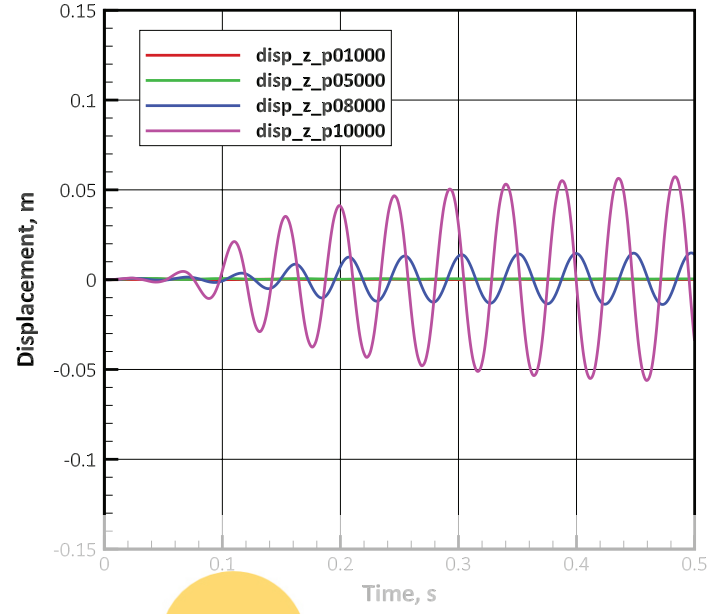

(a) freestream Mach number of 0.9 .

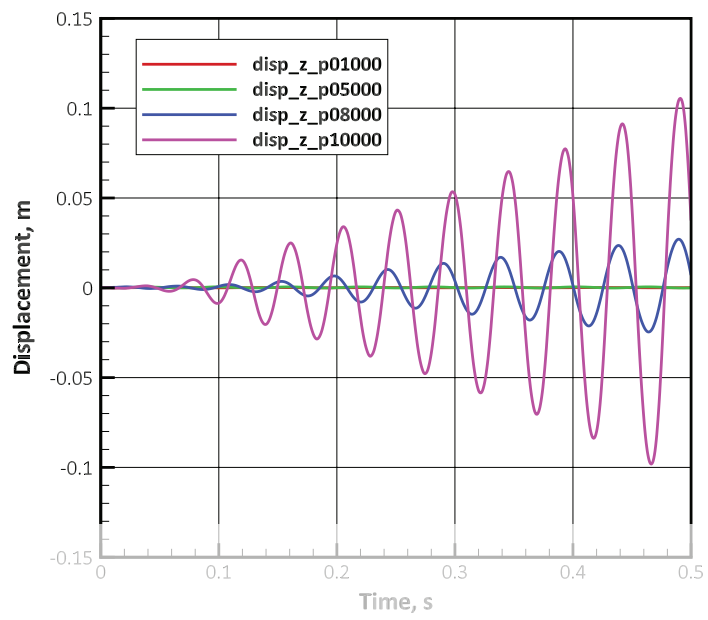

(b) freestream Mach number of 1.1 .

Figure 4: History of displacement in z-direction of wing tip.

observed in addition to the $22 \mathrm{~Hz}$ peak. However, it can be inferred from the displacement history that the $64 \mathrm{~Hz}$ single-end fixed delta the self-induced oscillation correspond to the eigenfrequency. Therefore, the coincidence of the characteristic frequen
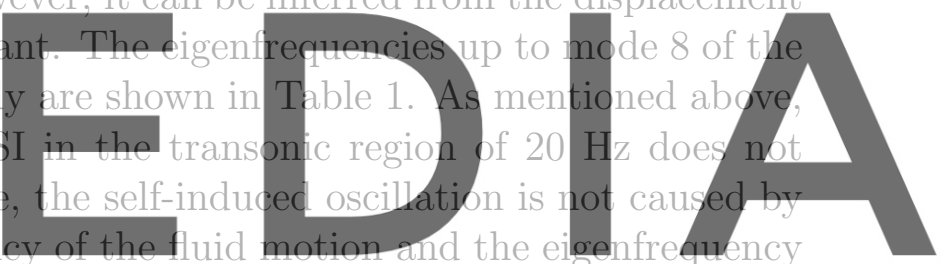

of the object, but is considered to be caused by another mechanism.

Register for free at https//www.scipedia.com to download the version without the watermark

Table 1: Eigenfrequency of the present delta wing.

\begin{tabular}{cc}
\hline Mode & Frequency, $\mathrm{Hz}$ \\
\hline 1 & 9.62 \\
2 & 36.69 \\
3 & 51.22 \\
4 & 88.94 \\
5 & 122.40 \\
6 & 158.89 \\
7 & 181.68 \\
8 & 217.62 \\
\hline
\end{tabular}

The time series distributions of Mach number near the delta wing and pressure coefficient at the delta wing surface for the case of freestream Mach number of 1.1 and reservoir 


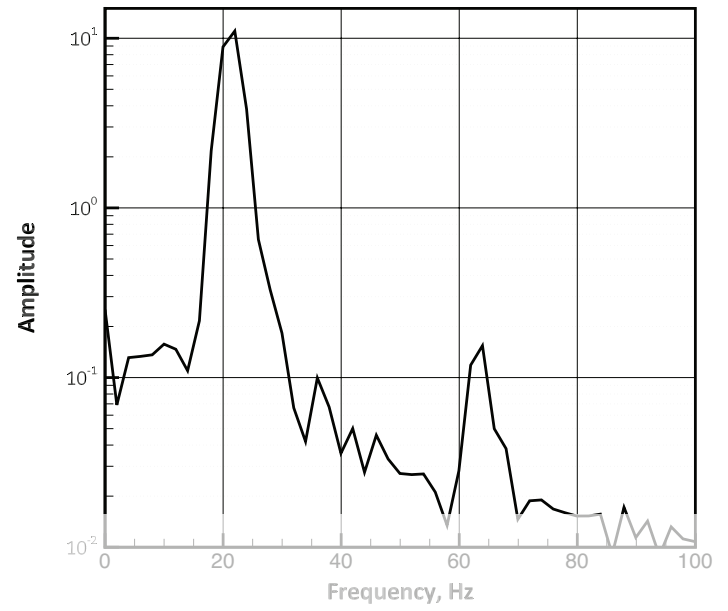

Figure 5: FFT result for displacements history for the case of Mach number of 1.1 and reservoir pressure of $10000 \mathrm{~Pa}$.

pressure of $10000 \mathrm{~Pa}$ are shown in Fig. 6. The Mach number distribution is for the $y=0.15$

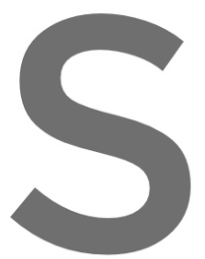
$m$ section of the comp right side to the left sick $6(\mathrm{a}), 6(\mathrm{~b}), 6(\mathrm{c})$, and 6 displacement velocity imum displacement wit displacement at this time, respectively ( de. These are for times $\mathbf{t}=0.429,0$,
(d) show the times of almost minin
in the $\mathbf{z}$ direction, maximum displac
ith negative displacement velocity i
ime, respectively (See also Fig. freestream is flowin
$41,0.454$ and 0.466
um displacenent wit
ment at this time, al
the $\mathrm{z}$ direction, and g from the
66 s. Figures
alth positive
almost min-

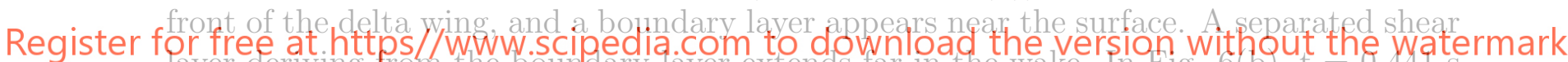
layer deriving from the boundary layer extends far in the wake. In $\mathrm{H} 1 \mathrm{~g}$. 6(b), t = o.441s

is the time when the displacement in the z-direction reaches its maximum in the positive

direction. Shock waves are generated on the upper surface of the wing in the positive $\mathrm{z}$-direction, while no shock waves are generated on the lower surface. At $\mathrm{t}=0.466 \mathrm{~s}$ in Fig. 6(d), the time when the displacement in the z-direction reaches its maximum in the negative direction. Shock waves are generated on the lower surface of the wing, while they do not appear on the upper surface of the wing.

The background of the increase in the amplitude of the self-induced oscillation is considered to be the aerodynamic force that drives it. Figure 7 shows the time histories of the z-direction displacement of the wing tip and the lift force acting on the wing. For comparison, the axes of the lift coefficient are reversed. The freestream conditions are Mach number 1.1 and reservoir pressure $10000 \mathrm{~Pa}$. As the relationship between displacement and lift, a profile like van der Pol oscillator appears. Such a relationship is also confirmed by the pitch instability in the transonic region of the Hayabusa sample return capsule [12].

In the case of a thin wing in the transonic region of this study, when the initial dis- 


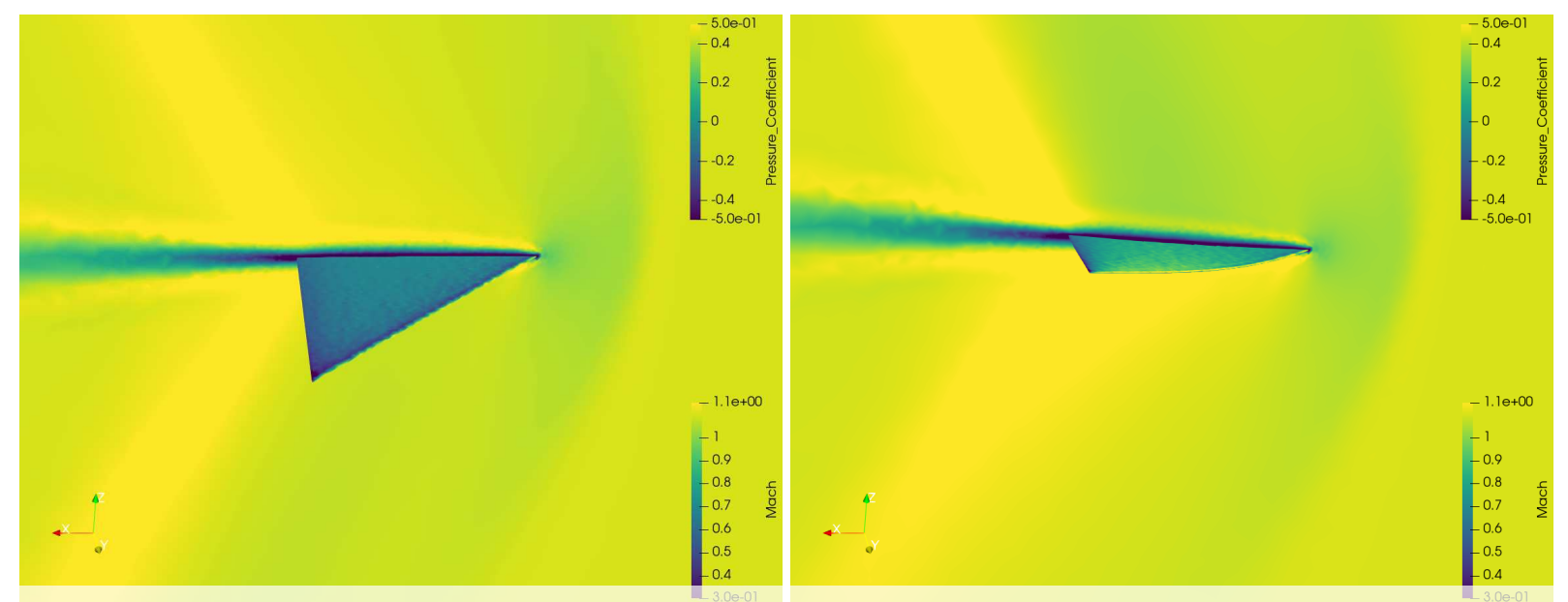

(a) $t=0.429 \mathrm{~s}$ (Minimum displacement).

(b) $\mathrm{t}=0.441 \mathrm{~s}$ (Maximum displacement).
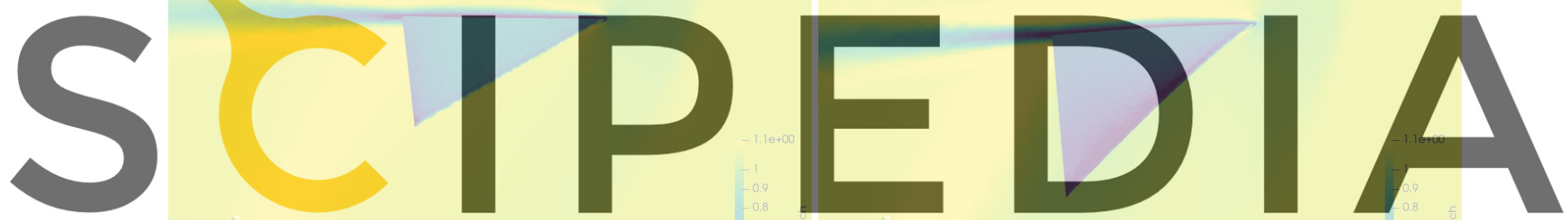

Register for free at https//www.scipedia.com to download the version without the watermark

(c) $\mathrm{t}=0.454 \mathrm{~s}$ (Minimum displacement).

(d) $\mathrm{t}=0.466 \mathrm{~s}$ (Maximum displacement).

Figure 6: Distributions of Mach number near wing and pressure coefficient on wing surface for the case of Mach number of 1.1 and reservoir pressure of $10000 \mathrm{~Pa}$.

turbance causes oscillation, shock waves are generated asymmetrically on the upper and lower surfaces of the wing. The high-pressure region behind the shock wave on one side of the wing tries to suppress the oscillation, while its response is accompanied by a slight delay, which acts as a phase delay of the external force against the oscillation. It is indicated that this is switched between negative and positive damping depending on the amount of displacement, resulting in self-induced oscillation. Therefore, the local elastic deformation produced by the shock wave plays a major role in the instability of the thin wing in the transonic region. In other words, the tight coupling between aerodynamics and elasticity has a large influence on the self-induced oscillation. 


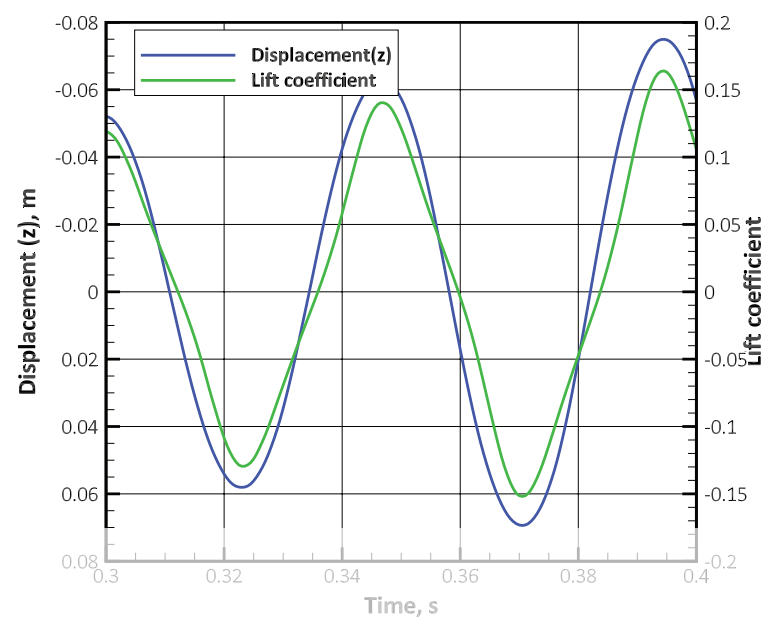

Figure 7: Histories of displacement of wing tip and lift coefficient for the case of Mach number of 1.1 and reservoir pressure of $10000 \mathrm{~Pa}$.

\section{CONCLUSIONS}

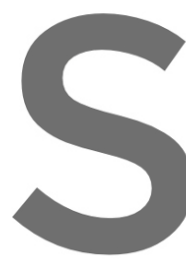

An open-source

been developed for

had been clarified.

compressible flow solver

element-based solver, and the
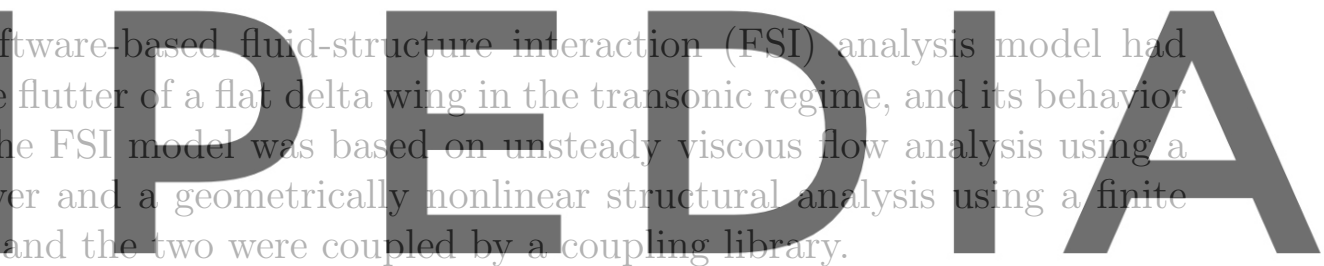

The FSI analysis showed that the self-induced oscillation is caused by the condition

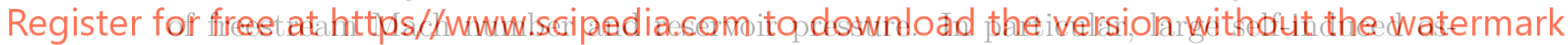

cillations occurred in the range of freestream Mach numbers 0.9 and 1.1. The oscillation

frequency was about $20 \mathrm{~Hz}$ regardiess of the freestream conditions. This suggested that the characteristic oscillation phenomenon of the flow does not cause unstable oscillation on the structure in the form of resonance, since there is no corresponding eigenfrequency of the present delta wing. In the case of significant self-induced oscillation, the shock wave on the wing surface formed a local high-pressure region. This induced a local elastic deformation of the wing, resulting in oscillation. The aerodynamic force acting on the wing against displacement was accompanied by a phase delay. The aerodynamic forces acted to promote the instability of the oscillation at small displacements, and acted as a damping mechanism at large displacements. These results suggested that, for an oscillation with a certain amplitude, the energy transfer between destabilization and stabilization reaches equilibrium within a cycle, and the oscillation eventually falls into a limit cycle. 


\section{ACKOWLEDGMENTS}

This work was supported by JSPS KAKENHI Grant Number 20H02360. This work was supported by "Joint Usage/Research Center for Interdisciplinary Large-scale Information Infrastructures" and "High Performance Computing Infrastructure" in Japan (jh200035).

\section{REFERENCES}

[1] Y. Takahashi and K. Yamada. "Aerodynamic heating of inflatable aeroshell in orbital reentry". Acta Astronautica, 152:437 - 448, 2018.

[2] Y. Takahashi, T. Koike, N. Oshima, and K. Yamada. "Aerothermodynamic analysis for deformed membrane of inflatable aeroshell in orbital reentry mission". Aerospace Science and Technology, 92:858-868, 2019.

[3] T.D. Economon, F. Palacios, S.R. Copeland, T.W. Lukaczyk, and J.J. Alonso. "SU2: An open-source suite for multiphysics simulation and design". Aiaa Journal, 54(3):828-846, 2016.

[4] G. Dhondt and K. Wittig. "Calculix a free software three-dimensional structural finite element program". www.calculix.de.

[5] H.J. Bungartz, F. Lindner, B. Gatzhammer, M. Mehl, K. Scheufele, A. Shukaev, and B. Uekermann. "preCICE-a fully parallel library for multi-physics surface coupling". Computers \& Fluids, 141:250-258, 2016.

[6] O.O. Bendiksen. "Review of unsteady transonic aerodynamics: Theory and applications". Progress in Aerospace Sciences, 47(2):135-167, 2011.

[7] "preCICE". https://github.com/precice.

[8] A. Jameson. "Origins and further development of the Jameson-Schmidt-Turkel scheme". AIAA Journal, 55(5):1487-1510, 2017.

[9] G. Karypis, K. Schloegel, and V. Kumar. "Parmetis: Parallel graph partitioning and sparse matrix ordering library". 1997.

[10] I. Miranda, R.M. Ferencz, and T.J.R. Hughes. "An improved implicit-explicit time integration method for structural dynamics". Earthquake Engineering 83 Structural Dynamics, 18(5):643-653, 1989.

[11] J. Degroote, P. Bruggeman, R. Haelterman, and J. Vierendeels. "Stability of a coupling technique for partitioned solvers in FSI applications". Computers 8 Structures, 86(23-24):2224-2234, 2008. 
[12] K. Hiraki. "Transonic Dynamic Instability of disk-shaped Capsule". The Institute of Space and Astronautical Science report. SP Aerodynamics, Thermophysics, Thermal Protection, Flight System Analysis and Design of Asteroid Sample Return Capsule, 17:265, 2003. 